\title{
Essential Aspects of Leprosy Classification and Clinical Course of Five Leprosy Patients, Including Four Relapsed Cases
}

\author{
Namisato $\mathrm{M}^{1 *}$ and Ogawa $\mathrm{H}^{2}$ \\ ${ }^{1}$ Department of Dermatology, Auen Poly-Clinic, Japan \\ ${ }^{2}$ Chief Director of Juntendo University, Japan
}

*Corresponding author: Masako Namisato, Director of Auen Poly-Clinic, 1037-1 Nakatomi Tokorozawa-city, Saitama-prefecture, 359-0002 Japan, Tel: +81-4-29905818; Fax: +81-4-2990-5828; Email: namima@auen.jp

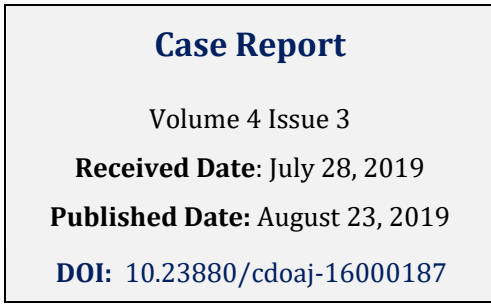

\section{Abstract}

The clinical course of leprosy varies depending on the level of host cell-mediated immunity to the causative agent, Mycobacterium leprae. In this study, the diagnostic aspects of the classification of the disease in five leprosy patients are presented alongside their clinical courses. The lepromatous leprosy (LL) Case presented with many erythematous plaques without anesthesia; pathology revealed the presence of abundant acid-fast bacilli (AFB). The patient developed reversal reactions during chemotherapy, and was diagnosed with subpolar LL. In this Case, resistance to three drugs was found. One borderline-tuberculoid (BT) leprosy Case had asymmetrically arranged multiple annular lesions, and another had one large erythematous lesion with small satellites. All these lesions were anesthetic, and the pathological findings revealed epithelioid granulomas; these clinicopathological findings are characteristic of BT leprosy. Another BT leprosy Case presented with neuralgia at the first visit, and on nerve biopsy, some nerve fascicles presented the feature of a reversal reaction. However, another nerve fascicle was found to be almost intact and showed positive staining in immunohistochemistry for M. leprae-specific phenolic-glycolipid I, although AFB were not found. In all three BT Cases, the slit-skin smears were negative. The borderline lepromatous (BL) leprosy Case involved numerous symmetric annular lesions with a characteristic punched-out feature. Loss of sensation was apparent in annular lesions. The smear tested positive, and abundant AFB were found inside the foamy Schwann cells. The differences in the clinical features of each patient reflect their immunity to the causative bacilli. In all, four Cases of the B-group were treated with the World Health Organization-recommended multibacillary regimen. One patient with BT leprosy developed anaphylactic shock against rifampicin (RFP). The monthly dose of RFP should therefore be administered following the direct observation procedure. The appropriate duration of chemotherapy for leprosy, especially in drug-resistant cases, should be studied further.

Keywords: Leprosy; Leprosy classification; Annular lesion; Reversal reaction; Chemotherapy 


\section{Clinical Dermatology Open Access Journal}

Abbreviations: LL: Lepromatous Leprosy; AFB: AcidFast Bacilli; BT: Borderline-Tuberculoid; BL: Borderline Lepromatous; RFP: Rifampicin; MB: Multibacillary; PB: Paucibacillary; LLp: Polar Lepromatous; TT: Polar Tuberculoid; DDS: Diaminodiphenyl Sulfone; AFB: AcidFast Bacilli; CAM: Clarithromycin; LVFX: Levofloxacin; MINO: Minocycline; PSL: Prednisolone; ENL: Erythema Nodosum Leprosum; BI: Bacterial Index.

\section{Introduction}

In the last 3 decades, new Cases of leprosy or Hansen's disease have become very rare in Japan. However, the few reported leprosy Cases were divided into 2 based on their backgrounds. The first group comprises individuals who were once cured with other regimens before the World Health Organization recommended the MDT [1] regimens; the second group also comprises migrants from various countries where leprosy is endemic.
Leprosy is a chronic granulomatous infection caused by Mycobacterium leprae (M. leprae). It is a model for understanding cell-mediated immunity in human beings. The clinical features of each patient directly reflect their immune status to causative bacilli.

The disease forms a spectrum, in which the clinical manifestations correlate with levels of host cell-mediated immunity to M. leprae. The 2 systems of classification, one is the simple one, which is the WHO-MDT [1,2] system composed of multibacillary (MB) and paucibacillary (PB); another is the Ridley system [3], which integrates the clinical and histologic changes in the patient, and is useful to understand leprosy. The system is a 6-membered spectrum, ranging from high to low resistance to $M$. leprae. These 2 classifications and MDT regimen are shown in the table. Although polar tuberculoid (TT) and polar lepromatous (LLp) are clinically stable, but between the poles, the host's granulomatous posture may change (Table 1).

\begin{tabular}{|c|c|c|c|c|c|c|}
\hline \multirow{2}{*}{ Ridley-Jopling system ${ }^{3}$} & Tuberculoid & \multicolumn{3}{|c|}{ Borderline } & \multicolumn{2}{|c|}{ Lepromatous } \\
\hline & TT & BT & $\mathrm{BB}$ & $\mathrm{BL}$ & LLs & LLp \\
\hline WHO-MDT system² & PB & \multicolumn{5}{|c|}{$\mathrm{MB}$} \\
\hline
\end{tabular}

TT: polar tuberculoid; BT: borderline tuberculoid; BB: Borderline; BL: Borderline Lepromatous; LL: Lepromatous Leprosy; LLs: Subpolar LL; LLp; polar LL; I: Indeterminate (early lesion without granuloma); PB: Paucibacillary; MB: Multibacillary WHO-MDT system: The classification for World Health Organization recommended multi-drug therapy

\begin{tabular}{|c|c|c|c|c|}
\hline Classification & $\begin{array}{c}\text { Number of skin lesions } \\
\text { Skin smear }\end{array}$ & Monthly dose & Daily dose & $\begin{array}{c}\text { Duration of } \\
\text { treatment }\end{array}$ \\
\hline PB & $<5 /$ negative & RFP 600mg & DDS 100mg & 6 months \\
\hline \multirow{2}{*}{ MB } & $\geqq 6 /$ positive & RFP 600mg & DDS $100 \mathrm{mg}$ & \multirow{2}{*}{12 months } \\
\cline { 2 - 4 } & & CLF 300mg & CLF 50mg & \multirow{2}{*}{} \\
\hline
\end{tabular}

MDT regimen (WHO-MDT) (1997)2

RFP: Rifampicin; CLF: Clofazimine; DDS: Diaphenylsulfone.

Table 1: Classification of Leprosy.

When the host's position in the spectrum moves toward a posture of higher resistance, it means upgrading or reversing, abrupt inflammatory symptoms, called reversal reaction, can develop [4]. It usually occurs after treatment initiation, or is sometimes observed at the time of diagnosis. We must always be cautious about possible developments of reversal reactions, because most leprosy-caused disabilities are related to reversal reactions.

This report presents 5 leprosy Cases: 1 Case of LLs, 3 Cases of BT, and 1 Case of BL. Each represents the essential aspect of each type of leprosy.
Namisato $\mathrm{M}$ and Ogawa H. Essential Aspects of Leprosy Classification and Clinical Course of Five Leprosy Patients, Including Four Relapsed Cases. Clin Dermatol J 2019, 4(3): 000187.

\section{Case presentation}

\section{Case 1}

A Japanese man aged 68 years during the first examination

He was diagnosed with leprosy when he was 16 years of age. He underwent leprosy treatment with sulfones until he turned 27 years. Six years later, his disease relapsed; diaminodiphenyl sulfone (DDS) and rifampicin (RFP) were prescribed, and later ofloxacin was also added. The details of these regimens are not clear. He entered remission approximately at the age of 43 years. 


\section{Clinical Features on the First Examination}

His face was red and edematous. The eyelids and ear lobes were swollen. Numerous red macules with varying sizes were disseminated and confluent on his trunk and extremities. Sensation in the skin lesions was normal. He found these lesions, which gradually increased, 10 months earlier. He had no visual problem. His right ulnar nerve was enlarged. Decreased eyelashes and eyebrows along with muscular weakness of his left hand have not changed during the last 20 years (Figure 1A).

The skin smear of red macules was $2+$ using the fluorescent staining method, equivalent to Gaffky 5. Two punch biopsies were taken from red macules on his abdomen. One was for pathological examination and other was subjected to drug-resistance assays.

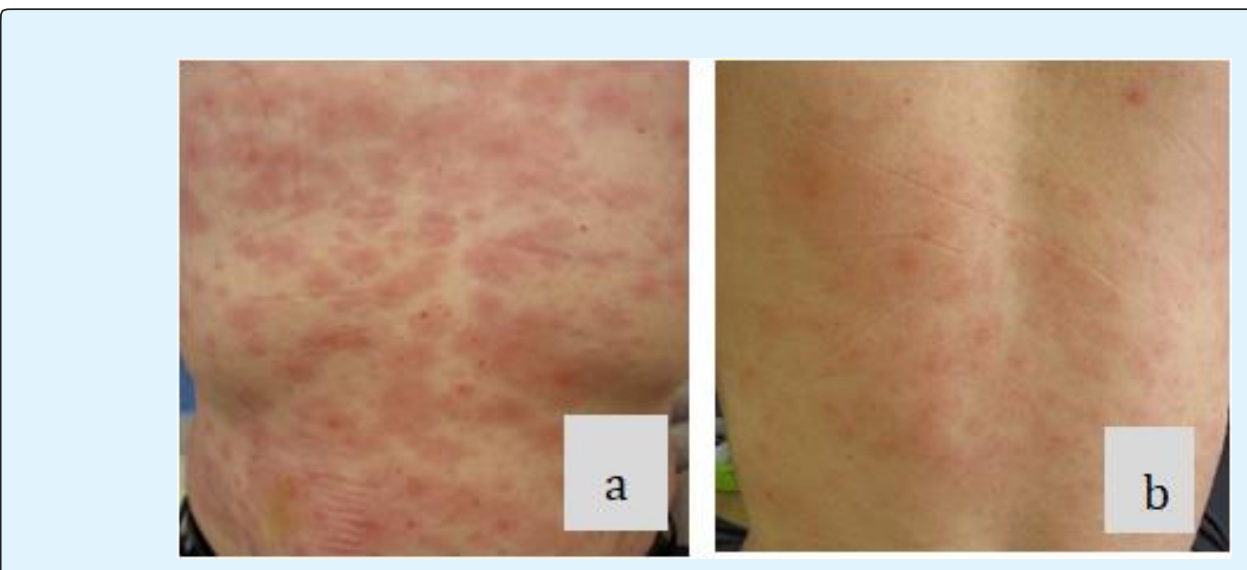

Figure 1A: Clinical feature. a,b: Numerous macules disseminated and confluent on the trunk.

Pathological Findings: Most parts of the dermis were occupied with proliferated foamy macrophages. Among these, there were a few lymphocytes. Some nerve bundles with slightly laminated perineurium were found in the cluster of macrophages. Many acid-fast bacilli (AFB) were found inside macrophages by Fite stain (Figure 1B).

He was diagnosed with lepromatous leprosy (LL).

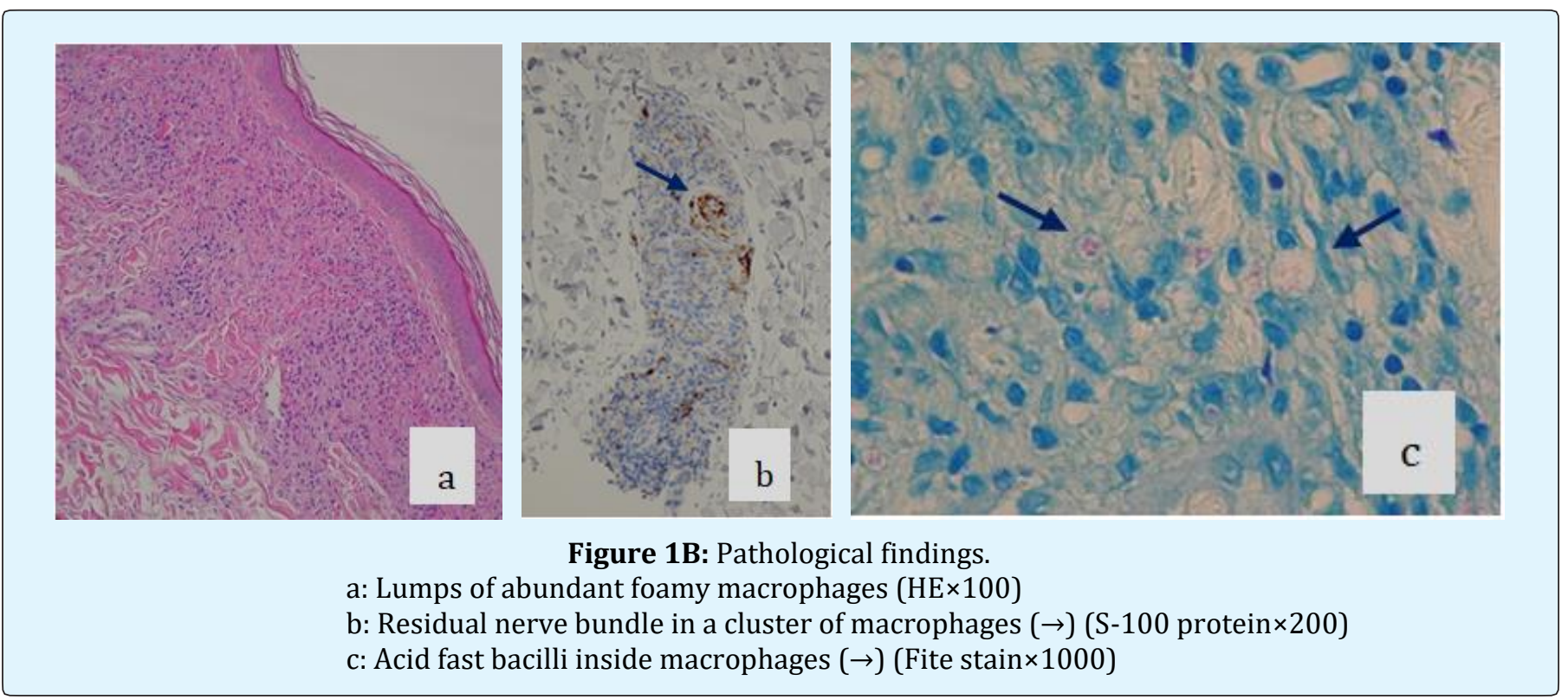


Treatment: Drug-resistance to DDS [folp1 55: CCC(Pro) $\rightarrow$ CGC(Arg)], RFP $[r p o B$ 456: TCG(Ser) $\rightarrow$ TTG (Leu)], and OFLX [gyrA 91: CGA(Ala) $\rightarrow$ GTA (Val)] was found. Therefore, a combination of minocycline (MINO), clarithromycin (CAM), and clofazimine (CLF) was started. CLF was given only for 15 days to avoid severe skin coloration. For the first 4 months, levofloxacin (LVFX) was also included considering possible intermixed new quinolone sensitive bacilli. MINO and CAM were given daily for about 1 year, then tapered to once weekly.

Two weeks after he started treatment, edematous redness on his face disappeared, and 5 months later, most skin lesions became flattened. The suddenly increased redness of previously existing lesions was noticed 1.5 years later. Then, similar symptoms repeated for 3 years. Approximately 3 years later, neuralgia of the median nerve also occurred. All these were compatible with a reversal reaction and controlled with a small dose of prednisolone (PSL).

About 5 years later, the outcome of the skin smear was negative, and treatment was ceased. Erythema nodosum leprosum (ENL) was not observed. He had no other medical problems. Based on all the clinical courses, he was diagnosed with LLs. Details of this Case are reported in the referenced paper [5].

\section{Case 2}

A Brazilian man aged 31 years during the first examination.

He found 1 annular lesion on his right lower leg 2 years ago; it grew larger and new lesions appeared 2 months before our first examination.

Clinical Features on the First Examination: Numerous well-defined red plaques of $1-4 \mathrm{~cm}$ in diameter and several larger annular lesions were asymmetrically disseminated on the face, trunk, and extremities. Loss of sensation, rough skin texture, and hair loss were apparent in these lesions. There was neither tenderness nor enlargement of the peripheral nerves. The skin smear was negative. Biopsy specimen was taken from the red macule on his left forearm (Figure 2A).

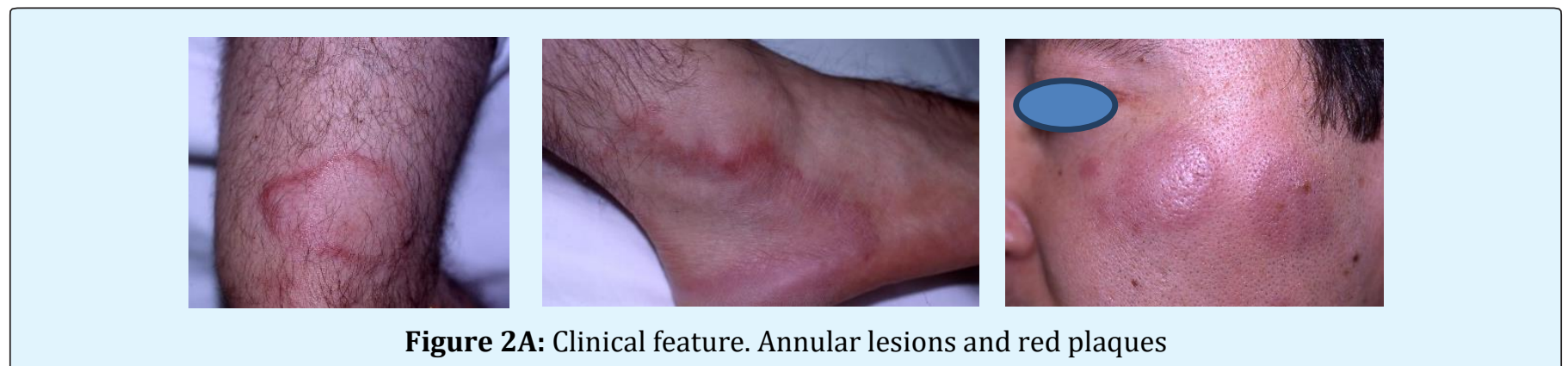

Pathological Findings: Degenerated nerve bundles, skin appendages, and clumped epithelioid cells were surrounded by densely infiltrated lymphocytes, forming well defined epithelioid cell granulomas. AFB was not found by Fite stain.

He was diagnosed with BT leprosy (Figure 2B).
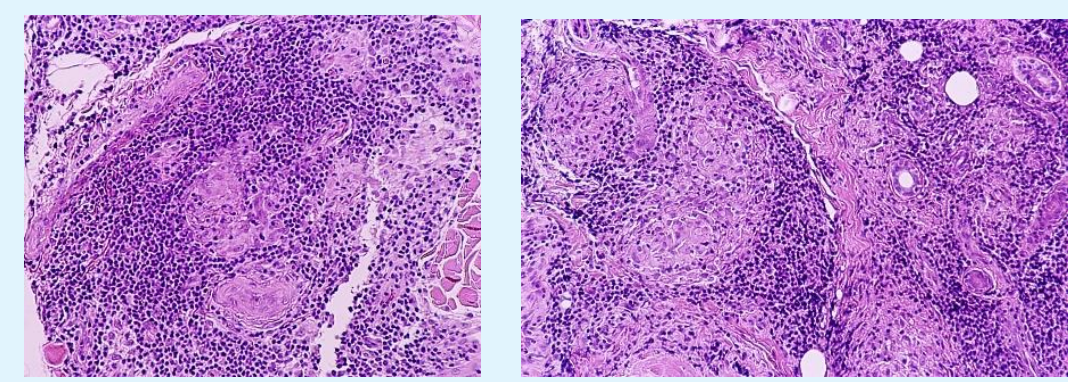

Figure 2B: Pathological findings. Degenerated nerve bundle, skin appendages and epithelioid cells were surrounded by numerous lymphocytes making granulomas $(\mathrm{HE} \times 200)$. 


\section{Clinical Dermatology Open Access Journal}

Treatment: WHO-MB's 1-year regimen was started. After completion of MDT, skin lesions disappeared leaving rough and hairless hypoesthetic lesions. No motor function impairment was noticed during the 3-year follow-up period.

\section{Case 3}

A Japanese man aged 64 years during the first examination

He was diagnosed with leprosy when he was 14 years of age, and cured with sulfone and RFP, but the details were unknown. He recognized skin lesions on his trunk 5 years before our first examination, then it gradually increased in its size.

Clinical Features on the First Examination: A huge erythema was seen on his light flank. The long diameter was around $30 \mathrm{~cm}$. It had well-defined slightly raised irregular borders. There were a few satellite lesions nearby. The huge skin lesion was anesthetic and anhidrotic. Peripheral nerves were neither tender nor enlarged. The skin smear was negative. The biopsy specimen was taken from the border of the large lesion
(Figure 3A).

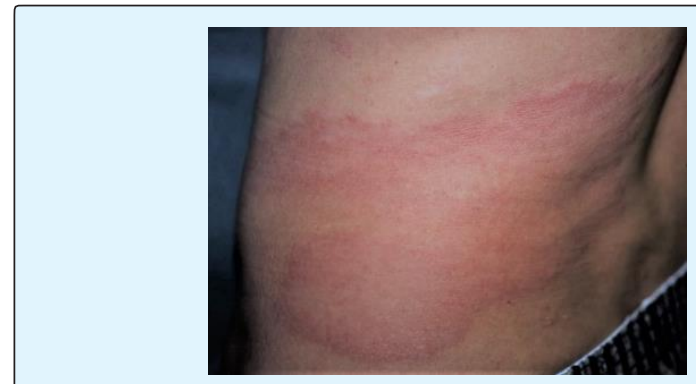

Figure 3A: Clinical feature. A huge erythema, having well defined but irregular border.

Pathological Findings: Skin appendages, nerve bundles, and epithelioid cells were tightly enclosed by densely infiltrated lymphocytes, making well-defined epithelioid granulomas. Multilayered perineurium of the small nerve bundle was also seen. No AFB was found by Fite stain (Figure 3B).

He was diagnosed with BT leprosy.

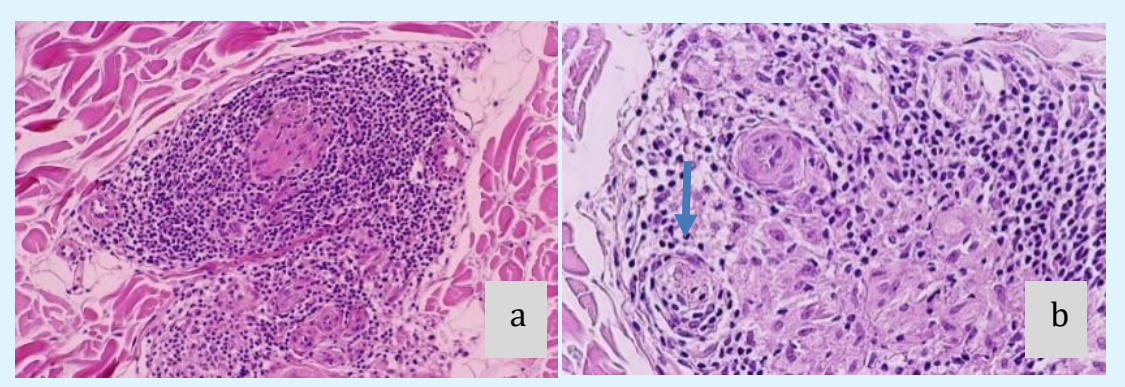

Figure 3B: Pathological findings. Skin appendages, nerve bundle and epithelioid cells are surrounded by many lymphocytes, making granuloma. Multilayered perineurium $(\rightarrow)(\mathrm{a}: \mathrm{HE} \times 100$, b: $\mathrm{HE} \times 200)$.

Treatment: The number of skin lesions was less than 6, but he had one very large lesion, therefore WHO-MB regimen was started. At the third monthly RFP administration, he developed anaphylactic shock. At this point, the skin lesions almost disappeared. Six months later, treatment with DDS and CLF was restarted and continued for 4 months. The details of this Case are reported in the referenced paper [6].

\section{Case 4}

A Brazilian man aged 39 years during the first examination

He found some skin lesions several years before in Brazil. He was diagnosed with leprosy, and a month's dose of MDT-PB was given. He only stopped treatment when the skin lesions disappeared. He experienced severe pain on both forearms, 2 weeks before our $1^{\text {st }}$ examination.

Clinical Features on the First Examination: Thickening of the left great auricular and common peroneal nerves were found. Right ulnar and median nerves were enlarged with tenderness. Several hypopigmented macules of less than $10 \mathrm{~cm}$ in diameter were also present. These lesions were dry and hypaesthetic. Muscle power of both fingers was slightly decreased. The skin smear was negative. Biopsy specimens were taken from the left sural nerve and hypopigmented macule of right forearm (Figure 4A). 


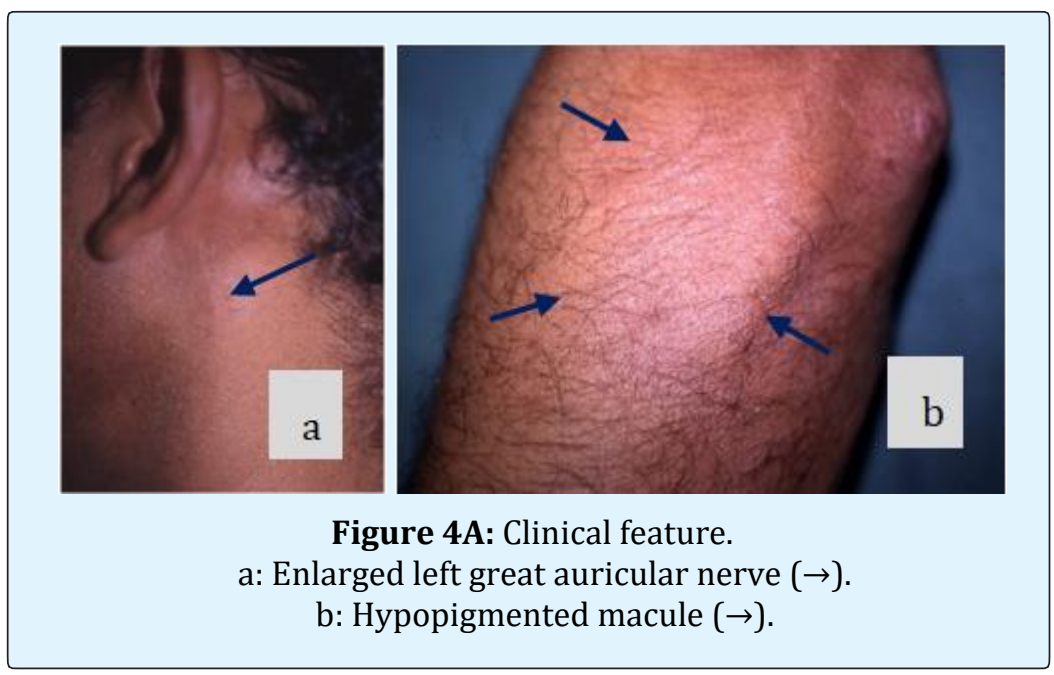

\section{Pathological Findings Skin biopsy}

Localized slight lymphocyte infiltration around sweat apparatuses and nerve fibers was seen in the upper dermis, with no granulomas. The feature was equivalent to indeterminate leprosy [7] (Figure 4B).

\section{Nerve biopsy}

Some nerve fascicles were destroyed and replaced by histiocytes, epithelioid cells, and lymphocytes accompanied with remarkable edema. However, small parts of the normal nerve tissue still remained. AFB was negative by Fite stain, but the remaining nerve tissue was stained positive in immunohistochemistry for M. lepraespecific phenolic glycolipid-I (PGL-I) [8] (Figure 4C).

He was diagnosed with BT leprosy accompanied with a reversal reaction. However, the skin lesion was compatible with indeterminate leprosy [7].

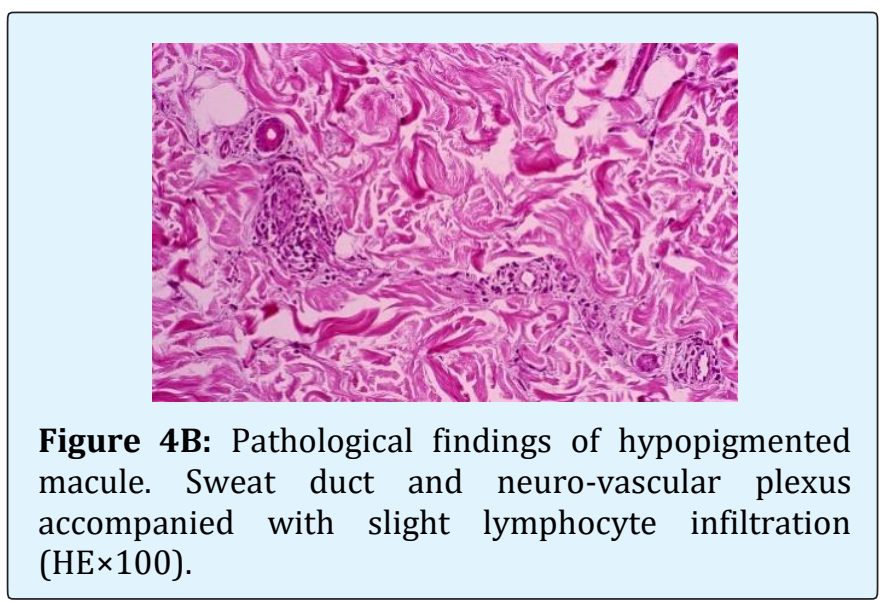

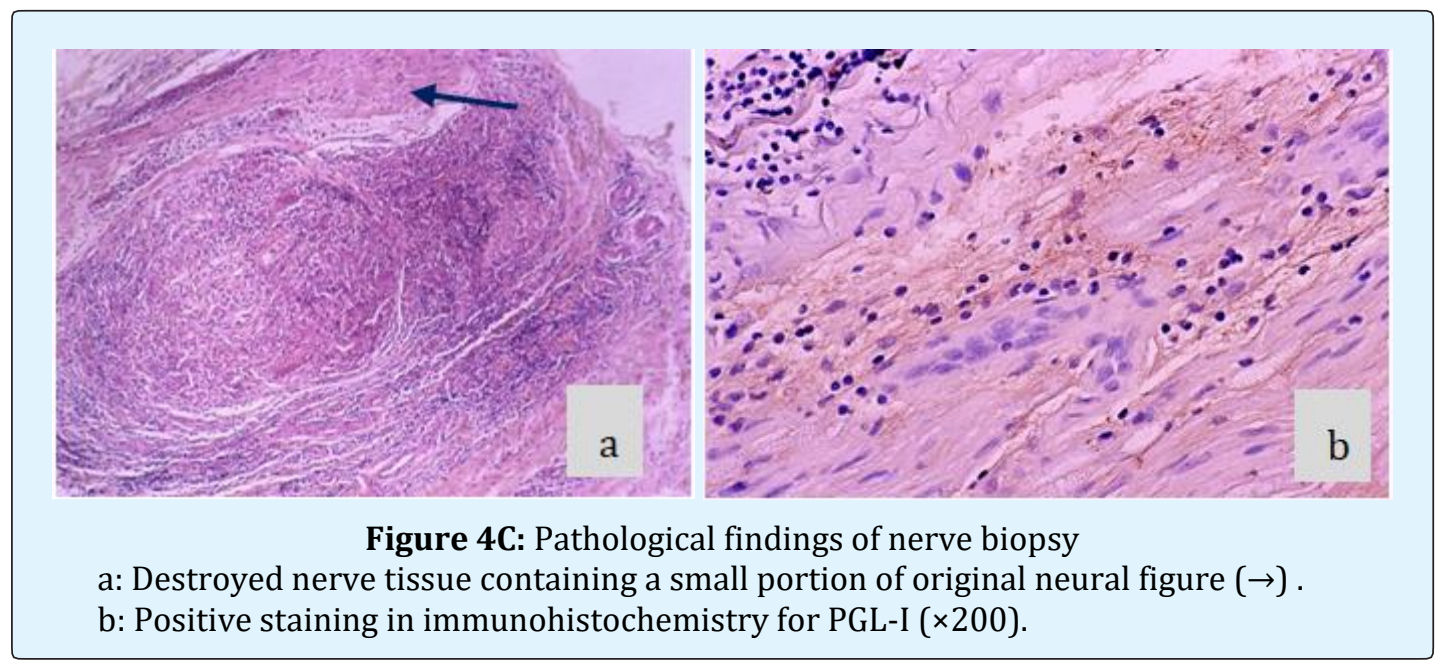




\section{Clinical Dermatology Open Access Journal}

Therapy: The number of thickened nerve trunks and skin lesions was more than 6; therefore, MDT-MB was started along with PSL of $20 \mathrm{mg}$ daily. The reversal reaction continued for 9 weeks. After completion of MDT-MB for 12 months, several anesthetic lesions were noticed on his extremities, but there was no apparent motor disfunction. Seven years later, we confirmed there were no active symptoms of leprosy.

\section{Case 5}

A Japanese man aged 66 years during the first examination

He was diagnosed with leprosy when he was 25 years of age; he was treated with sulfones and cured at about 48 years of age. He found 1 skin lesion 7 years before our first examination. Over time it became large and many other lesions developed. During this period, he had been taking small doses of DDS.

Clinical Features on the First Examination: Various sized annular lesions and erythematous macules disseminated on almost all his body. Some of them were arranged somewhat symmetrically. The inner borders of annular lesions were sharply marginated, but the outer borders had poorly marginated sloping edges; hence, dimorphic configurations or punched-out lesions were noticed. Most of the skin lesions were hypesthetic, and the insides of the annular lesions were anesthetic. The bacterial index (BI) [9] of the skin smear in red macule presented BI $(4+)$. Both sides of the ulnar nerve were enlarged and tender. Biopsy specimen was taken from the annular lesion on the abdomen (Figure 5A).

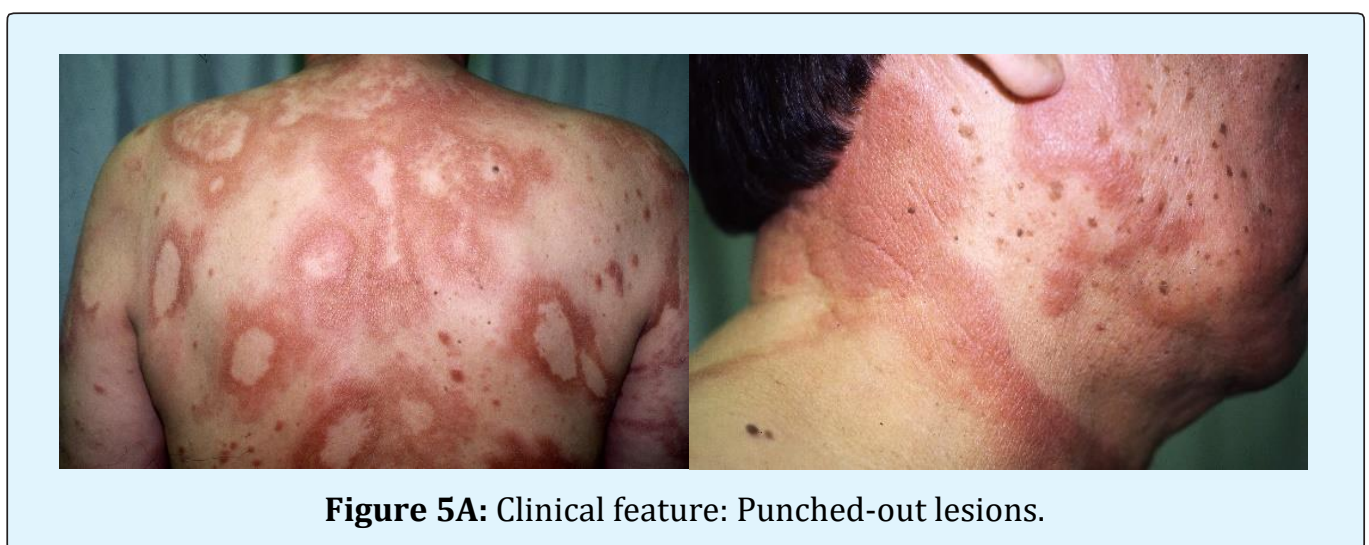

Pathological Findings: Disarranged nerve fibers and foamy epithelioid cells were surrounded by thick lymphocyte infiltration, making granulomas. Abundant
AFB, mostly fragmented, was found inside the vacuolated foamy Schwann cells (Figure 5B).

He was diagnosed with BL leprosy.

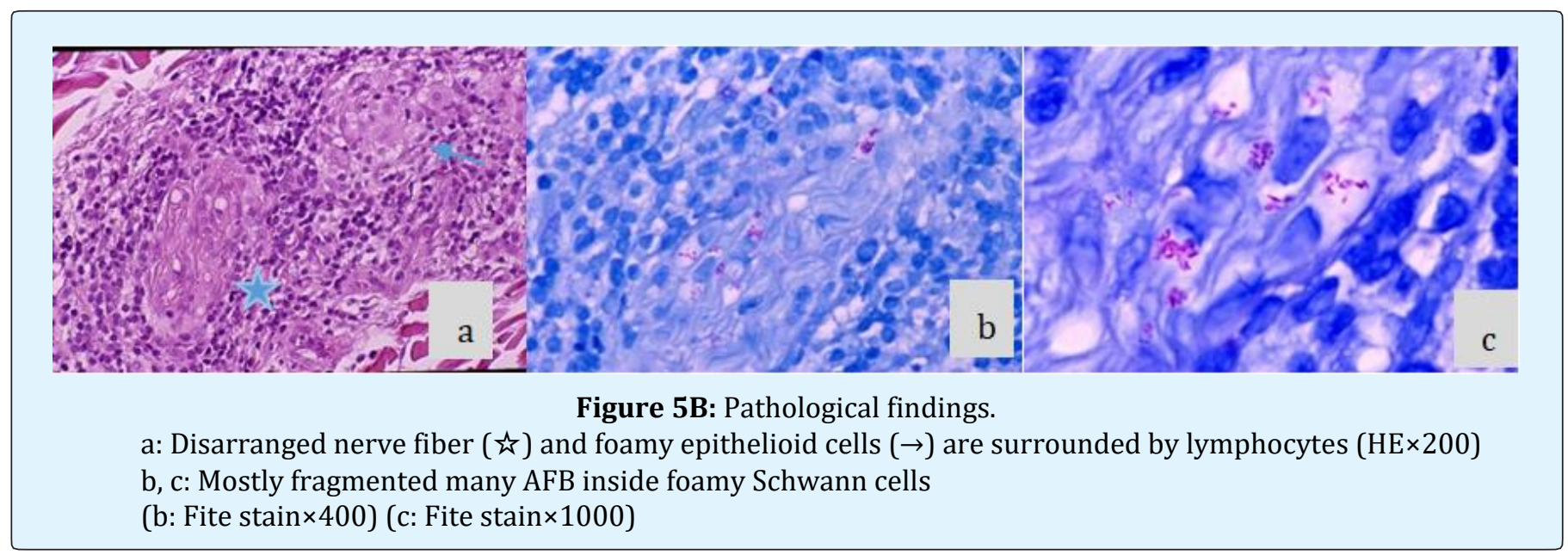


Treatment: After starting the MDT-MB regimen, bilateral, ulnar and median nerves became swollen with severe pain and tenderness. Most skin lesions presented rapidly increasing redness and tumidity. These were typical sign of a reversal reaction. PSL of $30 \mathrm{mg} /$ day was administrated then gradually tapered. After 10 months, these acute inflammatory symptoms subsided. MDT-MB regimen was given for 2 years, when BI (0) was achieved. About 10 years later, there was no active sign of leprosy.

\section{Discussion}

At the first examination of Case 1, the clinical characteristics presented as typical LL but couldn't be clarified as LLs or LLp. After starting chemotherapy, the patient developed a reversal reaction, which made it clear that his disease was LLs. In this Case, resistance to three drugs was noted, which is a problem for leprosy treatment. Although there is no guideline for Cases where there is triple-drug resistance to RFP, OFLX, and DDS, recently the WHO recommended CAM, MINO, and CLF for the patients with resistance to RFP and OFLX for 6 months, followed by CAM or MINO plus CLF for additional 18 months [10]. Fortunately, Case 1 was cured with MINO, CAM, and short-time administration of CLF and NQ, however further discussion is required for determining the ideal treatment protocol for multi-drug resistant Cases.

Case 2 and 3 presented characteristic skin lesions as seen in BT leprosy. These lesions, if there are many, are distributed asymmetrically. On the other hand, in BL leprosy, some skin lesions can be symmetrical.

Although the number of skin lesions in Case 3 was less than 6 , the big skin lesion which had an irregular border is considered to be caused by insufficient host immunity to M. leprae; usually, skin lesions in TT leprosy are less than $10 \mathrm{~cm}$.

Case 4 had two different conditions; indeterminate and BT leprosy concurrently. On the nerve biopsy, AFB was not seen by Fite stain, but immunohistochemistry staining for PGL-I was positive. The outermost layer of $M$. leprae is thickly covered by PGL-I, which remains long after the disappearance of M. leprae in a particular tissue. It is therefore supposed that M. leprae once invaded into the nerve tissue of Case 4.

The clinical course of BT leprosy can vary greatly. If any patient has neurological symptoms at the beginning of treatment (Case 4), careful management is necessary to prevent irreversible deformity caused by the reversal reaction.

The punched-out lesion seen in Case 5 is a diagnostic sign of BL leprosy. The pathological findings of epithelioid cells and coexisting foamy Schwann cells containing a large number of bacilli explain the downgrading tendency; it means that the patient was moving toward LL posture, but that he still had some host immunity to $M$. leprae.

The reversal reaction is not rare in the treatment of leprosy, except in the Cases of TT and LLp. It usually develops after treatment, though sometimes the patient may have already experienced this phenomenon at the first examination. The characteristic features of a reversal reaction include abrupt inflammation of previously existing lesions, accompanied with nerve swelling and neural pain. Histologically, an increase in lymphocytes, epithelioid cells, and giant cells are seen alongside severe edema. Cases 1, 4, and 5 presented some of these phenomena.

Case 4 was diagnosed with BT leprosy having a reversal reaction at the 1st examination. However, clinical manifestations of immunologically unstable B-group leprosy are considered to be equivalent to a reversal reaction. It may sometimes be difficult to clearly differentiate between BT leprosy and reversal reaction.

Anaphylactic shock caused by RFP, as seen in Case 4, tends to occur in intermittent regimens. Because of this possibility, monthly administration of RFP should be conducted under direct observation.

\section{Acknowledgment}

We gratefully acknowledge the kind support of Dr. Matsuoka M and Dr. Kai M of the Leprosy Research Center, National Institute of Infectious Disease, who kindly conducted the drug-resistance assay of Case 1 . We also really appreciate Dr. Izaki S for his useful opinions.

Conflict of Interests: Authors received no specific grants from any funding agency.

\section{References}

1. WHO Study Group (1982) Chemotherapy of Leprosy for Control Programmes. World Health Organization Technical Report Series 675: 1-33. 


\section{Clinical Dermatology Open Access Journal}

2. WHO Expert Committee on Leprosy (1998) Seventh Report. Technical Report Series 874. Geneva.

3. Ridley DS, Jopling WH (1966) Classification of leprosy according to immunity. Int J Lepr 34(3): 255-273.

4. Yawalkar SJ (1994) Reactions in Leprosy. In: Yawalkar SJ (Eds.), Leprosy. 6th (Edn.), CIBA-GEIGY Limited, Basle, Switzerland, pp: 83-91.

5. Namisato M, Fujiwara T (2018) Two Cases of leprosy who had drug resistance related mutations to diaphenylsulfone, rifampicin and new quinolone. Jpn J Lepr 86(3): 181-187.

6. Namisato M, Ogawa H (2000) Serious side effects of rifampin on the course of WHO/MDT, a Case report. Int J Lepr 68(3): 277-282.
7. Khanolkar VR (1964) Chapter 8, Pathology of leprosy. In: Cochrane RG (Eds.), Leprosy in Theory and Practice. $2^{\text {nd }}$ (Edn.), Davy TF, Bristol, John Wright and Sons LTD, pp: 125-126.

8. Hunter SW, Fujiwara T, Brennan PJ (1982) Structure and antigenicity of the major specific glycolipid antigen of Mycobacterium leprae. J Biol Chem 257(24): 15072-15078.

9. Ridley DS, Hilson GRF (1967) A logarithmic index of bacilli in biopsies I. Method. Int J Lepr 35(2): 184-186.

10. WHO Guidelines for the diagnosis, treatment and prevention of leprosy. 2018. 